\title{
Ocular Manifestations of Cytomegalovirus (CMV) - A Case Report
}

\author{
Sohan Lohiya ${ }^{1}$, Rajiv Pardasani² \\ 1, 2 Department of Ophthalmology, Jawaharlal Nehru Medical College, Datta Meghe Institute of Medical Sciences \\ (Deemed to Be University), Sawangi (Meghe), Wardha, Maharashtra, India.
}

\section{INTRODUCTION}

Cytomegalovirus (CMV) is a double-stranded DNA virus of the Herpesviridae family. ${ }^{1}$ Cytomegalovirus retinitis (CMVR) has been the most common opportunistic ocular infection and the leading cause of visual loss in acquired immunodeficiency syndrome (AIDS) patients representing about $90 \%$ cases of all infectious retinitis in this patient population. ${ }^{2}$

CMV retinitis is a common opportunistic ocular infection in patients with AIDS, representing reactivation of latent infection in macrophages and dendritic cells, and capable of reactivation if and when the cellular immune status is compromised, as in transplant recipients or patients with AIDS. ${ }^{1}$

CMVR is usually a clinical marker of immune deficiency. It tends to occur late in the course of the disease, and is associated with absolute cluster of differentiation 4 (CD4) $+\mathrm{T}$ lymphocyte counts below 50 cells / $\mu \mathrm{l}$. Anterior segment manifestations are due to anterior chamber associated immune deviation that enables the virus to survive in the anterior uveal tract. ${ }^{1}$

The symptoms of CMV retinitis depend on its initial location. Visual symptoms of peripheral lesions include floaters and loss of peripheral vision. Posteriorly located lesions involving macula or optic nerve, result in paracentral or central scotoma. The visual loss in CMV retinitis is usually the result of retinal necrosis and / or macular oedema. $^{3}$

\section{PRESENTATION OF CASE}

The patient is a 39-year-old male, Hindu by religion, and a farmer by occupation. The patient presented in the ophthalmology OPD with complaints of diminution of vision in both eyes which was gradual in onset and slowly progressive with floaters since 1 month.

The patient also complained of cough with expectoration since one and a half months. He also had fever ( 99 degree Fahrenheit) which was associated with chills with generalized weakness with cachexia for which he was referred to the medicine OPD. The patient was advised to get admitted in the medicine ward.

The patient is a known case of HIV since 2 years and was on antiretroviral therapy but then he became non-compliant to the medication. Past ocular and family history was unremarkable. There was no history of trauma or previous surgery.

After taking an informed verbal consent from the patient for examination and photographic documentation, a detailed ocular and systemic examination was carried out.

\author{
Corresponding Author: \\ Dr. Rajiv Pardasani, \\ F 16 Raghobaji Boys Hostel, \\ Datta Meghe Institute of Medical \\ Sciences (Deemed to Be University), \\ Sawangi (Meghe), Wardha, \\ Maharashtra, India. \\ E-mail: rajeev.pardasani@gmail.com
}

DOI: $10.14260 /$ jemds/2021/71

How to Cite This Article:

Lohiya S, Pardasani R. Ocular manifestations of cytomegalovirus (CMV) a case report. J Evolution Med Dent Sci 2021;10(05):319-322, DOI: $10.14260 /$ jemds $/ 2021 / 71$

Submission 22-10-2020,

Peer Review 05-12-2020,

Acceptance 10-12-2020,

Published 01-02-2021.

Copyright (C) 2021 Sohan Lohiya et al. This is an open access article distributed under Creative Commons Attribution License [Attribution 4.0 International (CC BY 4.0)] 


\section{General Examination}

The patient was conscious, co-operative \& well oriented.

The patient was thin built, poorly nourished.

The patient was febrile (99 degrees Fahrenheit)

Pulse was 90 beats / $\mathrm{min}$

Blood pressure was $130 / 90 \mathrm{~mm} \mathrm{Hg}$

The patient had generalized lymphadenopathy.

There was no pallor, icterus, cyanosis, clubbing, or pedal edema present

\section{Systemic Examination}

Cardiovascular system: Within normal limits.

Respiratory system: Right sided infraclavicular creps present, left sided dull note on percussion and decreased breath sound on auscultation.

Per-abdomen: Within normal limits.

Central nervous system: No gross sensory motor deficit elicited.

\section{Ocular Examination \\ Right Eye (OD)}

Uncorrected Visual Acuity: Counting finger close to face, perception of light present, projection of rays accurate in all quadrants. There was no improvement on pinhole and the best corrected visual acuity could not be assessed. Near vision and colour vision could not be assessed.

Intraocular pressure (IOP): $13 \mathrm{mmHg}$ by Goldmann applanation tonometer.

Eyebrows: Normal.

Ocular motility and alignment: Full and free in all directions of gaze.

Slit lamp exam:

Anterior segment

Eye Lids: Normal,

Conjunctiva: Mild conjunctival congestion,

Cornea: Bright.

Anterior chamber: Normal in depth.

Iris: Normal

Pupil: Single in number, 2 - $3 \mathrm{~mm}$ in size, circular in shape, the pupillary reaction was normal stimulus response to light (NSRL)

Lens: Normal.

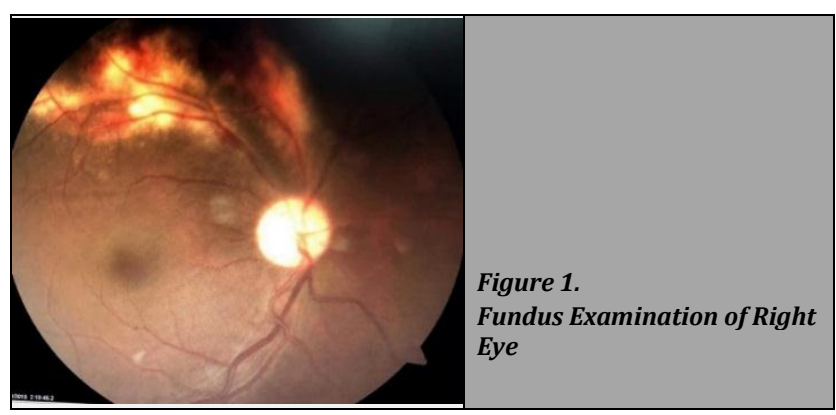

Dilated fundus examination (Figure 1) Right eye fundus revealed clear media; optic disc size normal, shape circular, all margins were blurred, disc colour was pale; cup disc ratio was obliterated, peripapillary area was normal, blood vessels were tortuous in all quadrants and foveal reflex was dull. There were intraretinal haemorrhages and exudates in superotemporal quadrant. Cotton wool spots were present in nasal, temporal to disc and in inferotemporal quadrant.

\section{Left Eye (OS)}

Uncorrected Visual Acuity: Counting finger close to face, perception of light present, projection of rays accurate in all quadrants. There was no improvement on pinhole and the best corrected visual acuity could not be assessed. Near vision and colour vision could not be assessed.

Intraocular pressure (IOP): $10 \mathrm{mmHg}$ by Goldmann applanation tonometer.

Eyebrows: Normal.

Ocular motility and alignment: Full and free in all directions of gaze.

Slit Lamp Exam

Anterior Segment

Lid: Normal

Conjunctiva: Mild conjunctival congestion

Cornea: Bright

Anterior chamber: Fine keratic precipitates, $1+$ aqueous cells with flare. Posterior synechiae was present in 360 degree.

Iris: Normal

Pupils: Single in number, 2 - $3 \mathrm{~mm}$ in size, circular in shape Lens: Normal.

There was relative afferent pupillary defect (RAPD) (grade 2 ) in the left eye on swinging flashlight test.

Dilated fundus examination (Figure 2) left eye fundus revealed clear media, optic disc size and shape could not be assessed, all margins were blurred, disc colour was pale; cup disc ratio was obliterated, blood vessels were tortuous in all quadrants and vascular sheathing was seen in the various clock area, foveal reflex was dull and exudates were present in the macular area surrounding fovea. There were intraretinal haemorrhages and exudates along the inferior arcade and inferotemporal quadrant.

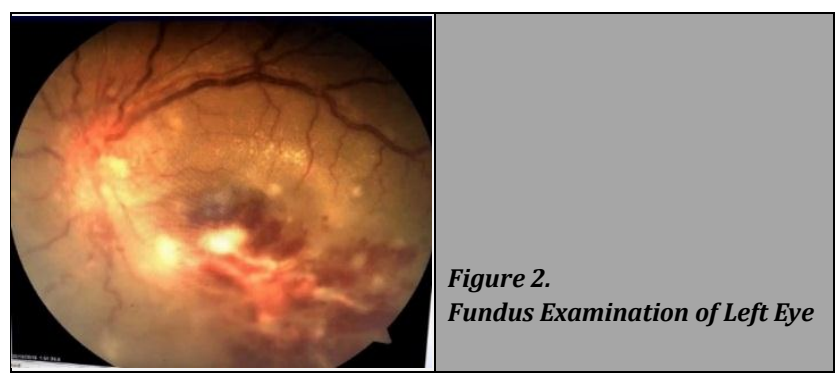

\section{Diagnostic Assessment}

The primary differential which was considered when the patient first presented was CMV retinitis. After consultation with the physician routine blood investigations were done which showed reduced haemoglobin (6 g \%), reduced red blood cell (RBC) count (2.55 million / $\left.\mathrm{mm}^{3}\right)$, raised erythrocyte sedimentation rate (ESR) (38 $\mathrm{mm}$ at the end of 1 $\mathrm{hr}$ ). Liver function test (LFT) was within a normal range. In kidney function test (KFT), sodium was deranged (128 mEq / l), rest were within normal range.

Chest x-ray posteroanterior (PA) view (Figure 3) was done which was suggestive of patchiness in pericardial regions and left middle zone. 
To confirm the finding of chest $\mathrm{x}$-ray, high resolution computed tomography thorax (Figure 4,5) was done which showed left-sided minimal pneumothorax, multiple cavitary lesions in right middle lobe with changes of cystic bronchiectasis, emphysematous changes in bilateral lung fields. Cluster of differentiation 4 (CD4) counts were 24 cells per $\mu$ l.
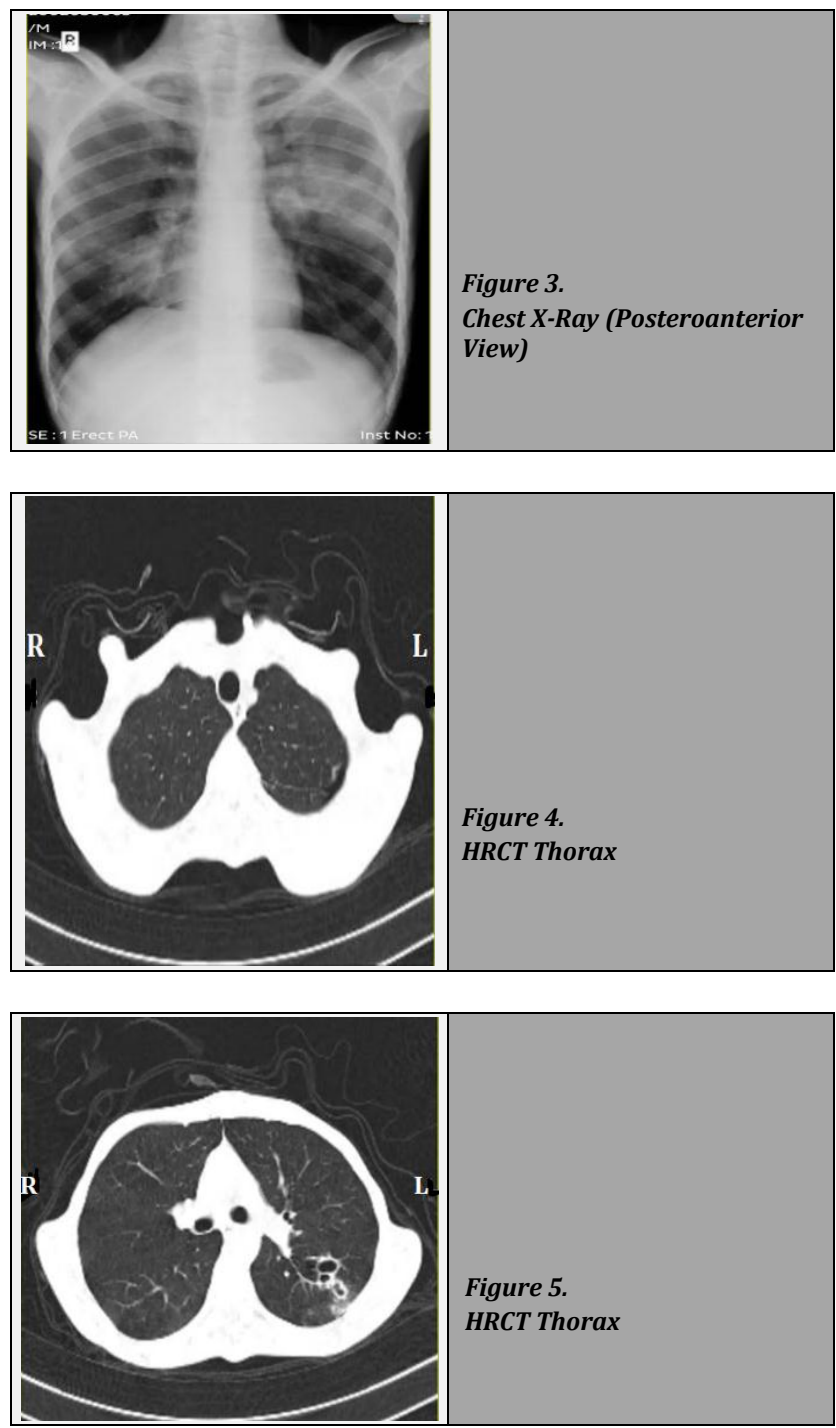

Vitreous biopsy was done by polymerase chain reaction technique which revealed high concentration of CMV DNA in the sample.

Diagnosis of "immunocompromised host with Pneumocystis carinii pneumonia" was made by physicians and treatment was started accordingly.

\section{DISCUSSION OF MANAGEMENT}

Before the intervention, the patient was transfused with 2 units of packed red cells and haemoglobin improved (11 gm $\%)$. The patient was started on antiretroviral therapy, antitubercular drugs and antibiotics. Intravenous ganciclovir $5 \mathrm{mg} / \mathrm{Kg}$ twice daily for 2 weeks followed by $5 \mathrm{mg} / \mathrm{Kg}$ once daily was advised. The patient was also counselled about the intravitreal injection, but the patient did not give consent for intravitreal injection.
The patient was observed in the medicine ward and was started on the following medications:-

Injection ganciclovir $5 \mathrm{mg} / \mathrm{Kg}$ twice a day for 15 days followed by $5 \mathrm{mg} / \mathrm{Kg}$ once a day for 11 days.

Capsule doxycycline $100 \mathrm{mg}$ twice a day for 10 days

Tablet clarithromycin $500 \mathrm{mg}$ twice a day for 5 days

Antitubercular drugs: Tab rifampicin (1 cap) $450 \mathrm{mg}$, tab isoniazid (1 tab) $300 \mathrm{mg}$, tab pyrazinamide (1 tab) $750 \mathrm{mg}$, tab ethambutol (1 tab) $800 \mathrm{mg}$ once daily.

Antiretroviral drugs: tab tenofovir $(300 \mathrm{mg})+\mathrm{tab}$ lamivudine (300 mg) + tab lopinavir (800 mg) once daily

Tablet sulfamethoxazole + trimethoprim twice a day for 20 days.

Tablet fluconazole for 25 days

Tablet pantoprazole $40 \mathrm{mg}$ once a day.

Syrup Grilinctus 2 teaspoon three times a day for 14 days

E / d moxifloxacin four times a day

E / d atropine $0.1 \%$ three times a day.

\section{Follow-Up and Outcomes}

After discharge, the patient was followed up after 1 month in both Medicine and Ophthalmology OPD.

The patient's haemoglobin, RBC count, WBC count, CD4 level were re-valuated, as ganciclovir causes hematological abnormalities (neutropenia, anaemia, and thrombocytopenia). ${ }^{4}$

Both eyes were examined which showed that uveitis had reduced in the left eye but the vision did not improve in both eyes. The pupil was fixed and dilated due to atropine and IOP was normal on follow up. Dilated fundus examination showed clear media, optic disc margins were blurred, pale disc, absent foveal reflex, intraretinal haemorrhages were resolved in the right eye and were reduced to few in number in the left eye along inferotemporal quadrant. Exudates were resolved.

\section{DISCUSSION}

The case highlights how a multidisciplinary approach and early recognition of symptomatic presentation can help in successful management and rehabilitation in the case of CMV retinitis.

HIV associated ophthalmic diseases arrays from adnexal disorders to posterior segment diseases, CMV infection being the most common worldwide. It accounts for $70-85 \%$ of CMV infections and is the leading cause of visual impairment and blindness. ${ }^{5}$ following primary infection, the virus spreads through haematogenous route and can infect the retina. ${ }^{6}$

The virus enters a non-replicative state. In the eye, the vascular endothelial cells of the retina may be the initially infected target. The deregulated cell-mediated immune response in AIDS patients leads to virus persistence and pathogenesis in the retina. ${ }^{7}$

CMV retinitis occurs usually as CD4 count decreases below $50 / \mathrm{mm} .{ }^{3.6}$ In our case, the CD4 count was 24 cells $/ \mathrm{mm}^{3}$ at the time patient presented to us. As optic atrophy had already set in, the vision did not improve. During the course of treatment, patient's uveitis was treated successfully.

CD4 counts indicates immune dysfunction and do not reflect functional abnormalities in the immune system. ${ }^{8}$ The incidence of CMV retinitis has declined dramatically among 
patients maintained on highly active antiretroviral therapy (HAART). Prior to the introduction of HAART, the natural history of untreated CMV retinitis was relentless progression of full thickness retinal necrosis leading to blindness. Some patients with CMV retinitis who respond to HAART in terms of elevated CD4 count and reduced HIV viral load undergo no reactivation of their retinitis despite having no specific antiCMV therapy. ${ }^{3}$

As there is increased access to HAART and increased survival of patients with AIDS, it is possible to witness a change in the pattern of CMV retinitis in HIV. Although the incidence of CMV retinitis has reduced with the introduction of HAART, CMV retinitis remains a threat to the vision in these patients. ${ }^{3}$ HAART combination is successful in significantly reducing the number of plasma HIV messenger ribonucleic acid (RNA) copies and in increasing the number of CD4 + T lymphocytes causing immune recovery. ${ }^{9}$ The treatment should be based on the degree of ocular involvement, retinal zone involvement, immune status, underlying medical conditions, access to medical care.

Ganciclovir is a selective viral DNA polymerase inhibitor, given with an induction dose for two to three weeks followed by lower maintenance doses to prevent relapse and is used as a first-line therapy intravenously. Oral ganciclovir has poor absorption and is used for maintenance or in combination with other therapy. In $80-90 \%$ of patients, intravenous (IV) administration of ganciclovir twice daily (induction therapy) (5 mg / Kg) for 21 days initially stops retinal-cell necrosis and reduces viral recovery from blood. After 2 - 3 weeks of induction therapy, patients are usually shifted to maintenance therapy. Dose adjustments of ganciclovir are done appropriately as per renal function as it causes neutropenia, anaemia, and thrombocytopenia. ${ }^{4}$

Valganciclovir, which is a prodrug of ganciclovir with high oral bioavailability (60\%). It is given as $900 \mathrm{mg}$ once daily for 2 to 3 weeks resulting in comparable serum ganciclovir levels achieved with daily intravenous induction. Valganciclovir is the drug of choice, due to its lower cost and high bioavailability. As here valganciclovir was not available so ganciclovir was used. ${ }^{4}$

In a study conducted by Martin DF et al., induction treatment with intravenous ganciclovir achieved $77 \%$ satisfactory response when a similar response was achieved in $72 \%$ who were assigned to oral valganciclovir. ${ }^{10}$

Intravitreal ganciclovir allows high doses in the eye and decreases systemic complications by allowing lower systemic concentrations. Intravitreal therapy should be preferred over intravenous therapy but here our patient did not give consent for intravitreal.

Patients must be re-examined every 1 to 2 months to determine retinitis status. If the recurrent disease develops, a change in therapy is indicated. Patients with a good response to HAART therapy may be considered for withdrawal from anti-CMV therapy after the CD4 + T lymphocyte count has been maintained at approximately 150 cells $/ \mathrm{mm}^{3}$ for 3 months. ${ }^{9}$ The increasing availability of HAART in countries such as India, may change the incidence of CMV retinitis due to several factors, including longer survival, improved immunologic status, and increased incidence of immune recovery uveitis. ${ }^{11}$

\section{CONCLUSIONS}

CMV retinitis has been a common infection in HIV patients. With the increasing availability of HAART, there has been an improvement in prognosis of HIV infected patients. This has drifted the usual pattern and incidence of CMV retinitis. CMV retinitis presents with bilateral visual loss and thus there is a need to emphasize on early screening strategies regardless of CD4 count. Treatment with intravenous therapy has shown effective results and oral medications, though with low efficacy are also given to aid in treatment. Appropriate maintenance dose should be given so as to avoid recurrence.

Financial or other competing interests: None.

Disclosure forms provided by the authors are available with the full text of this article at jemds.com.

\section{REFERENCES}

[1] Gross JG, Sadun AA, Wiley CA, et al. Severe visual loss related to isolated peripapillary retinal and optic nerve head cytomegalovirus infection. Am J Ophthalmol 1989;108(6):691-8.

[2] Gupta RP. Cytomegalovirus retinitis: a case report. Medical Journal of Dr. D. Y. Patil University 2014;7(6):822-4.

[3] $\mathrm{Au}$ Eong KA, Beatty S, Charles SJ. Cytomegalovirus retinitis in patients with acquired immune deficiency syndrome. Postgrad Med J 1999;75(888):585-90.

[4] Stewart MW. Optimal management of cytomegalovirus retinitis in patients with AIDS. Clin Ophthalmol 2010;4:285-99.

[5] Thorne JE, Jabs DA, Kempen JH, et al. Causes of visual acuity loss among patients with AIDS and cytomegalovirus retinitis in the era of highly active antiretroviral therapy. Ophthalmology 2006;113(8):1441-5.

[6] Karkhaneh R, Lashay A, Ahmadraji A. Cytomegalovirus retinitis in an immunocompetent patient: a case report. J Curr Ophthalmol 2016;28(2):93-5.

[7] Scholz M, Doerr HW, Cinatl J. Human cytomegalovirus retinitis: pathogenicity, immune evasion and persistence. Trends Microbiol 2003;11(4):171-8.

[8] Ang BC, Teoh SC. Case report of cytomegalovirus retinitis in an HIV-positive patient with a CD4-count nadir of 254 cells per $\mu$ l. Eye (Lond) 2012;26(8):1153-4.

[9] Sobrin L, Foster CS. Cytomegalovirus retinitis after one decade of HAART. Int Ophthalmol Clin 2007;47(2):15564.

[10] Martin DF, Sierra-Madero J, Walmsley S, et al. A controlled trial of valganciclovir as induction therapy for cytomegalovirus retinitis. $\mathrm{N}$ Engl J Med 2002;346(15):1119-26.

[11] Shah SU, Kerkar SP, Pazare AR. Evaluation of ocular manifestations and blindness in HIV/AIDS patients on HAART in a tertiary care hospital in Western India. Br J Ophthalmol 2009;93(1):88-90. 